Research Article

\title{
Clinicopathological Features and Increased Expression of Toll-Like Receptor 4 of Gastric Cardia Cancer in a High-Risk Chinese Population
}

\author{
Guangcan Chen, ${ }^{1}$ Muming Xu, ${ }^{2}$ Jingyao Chen, ${ }^{3}$ Liangli Hong $\mathbb{D D}^{4}{ }^{4}$ Wenting Lin, ${ }^{3}$ \\ Shukun Zhao $\mathbb{1 0},{ }^{3}$ Guohong Zhang $\mathbb{D},{ }^{3}$ Guo Dan, ${ }^{3}$ and Shuhui Liu $\mathbb{D}^{3}$ \\ ${ }^{1}$ Department of Gastrointestinal Surgery, The First Affiliated Hospital of Shantou University Medical College, Shantou, \\ Guangdong Province 515041, China \\ ${ }^{2}$ Department of Abdominal Surgery, The Tumor Hospital of Shantou University Medical College, Shantou, \\ Guangdong Province 515041, China \\ ${ }^{3}$ Department of Pathology, Shantou University Medical College, Shantou, Guangdong Province 515031, China \\ ${ }^{4}$ Department of Pathology, The First Affiliated Hospital of Shantou University Medical College, Shantou, \\ Guangdong Province 515041, China
}

Correspondence should be addressed to Shuhui Liu; liushuhuistu@126.com

Received 26 July 2017; Revised 14 November 2017; Accepted 28 December 2017; Published 18 February 2018

Academic Editor: Qing Lin

Copyright $\odot 2018$ Guangcan Chen et al. This is an open access article distributed under the Creative Commons Attribution License, which permits unrestricted use, distribution, and reproduction in any medium, provided the original work is properly cited.

The incidence of gastric cardia cancer (GCC) is high in China. However, the clinicopathological characteristics and the carcinogenesis of GCC are unclear. Toll-like receptor 4 (TLR4) is an important innate immunity receptor and has a role in nonGCC (NGCC). We compared the clinicopathological characteristics of GCC patients from a high-risk area in China to esophageal cancer (EC) patients. Immunohistochemistry for TLR4 was performed in 201 histological samples of normal gastric cardia mucosa $(n=11)$, gastric cardia inflammation $(n=87)$, and GCC $(n=103)$. We included 84 patients with EC and 99 with GCC. GCC tissue was more poorly differentiated than EC tissue and more invasive, with more histomorphologic variation. Lymph node metastasis was more frequent in GCC than in EC. The Helicobacter pylori infection rate was higher but not significantly with GCC than EC. Survival was shorter with lymph node metastasis. We found a statistically significant trend for progressive increase of TLR4 expression from normal mucosa to inflammation in GCC. GCC in this high-risk area displays clinicopathologic characteristics different from those of EC and different from those of gastroesophageal junction carcinomas in other countries, although this was not analyzed statistically. Increased TLR4 expression in gastric cardia lesions may be associated with GCC tumorigenesis.

\section{Introduction}

Gastric cancer is the fifth most common malignancy, with an estimated 952,000 new cases in 2012 worldwide [1]. Although gastric cancer is still a major contributor to the global cancer burden, its incidence has decreased over the past decades [1].

Generally, gastric cancers can be classified into two categories: gastric cardia cancer (GCC) arising in the area of the stomach adjacent to the gastroesophageal junction (GEJ) and non-GCC (NGCC) arising from more distal regions of the stomach [2]. The incidence and risk factors for GCC and NGCC vary considerably. The decreasing incidence of gastric cancer is due mostly to the declining trend of NGCC; however, the incidence of GCC may be increasing [3-5]. In addition to different epidemiology, GCC and NGCC are thought to have different risk factors, clinicopathologic 
features, and gene expression [6-8]. Thus, current research is addressing GCC and NGCC as separate diseases.

From the Cancer Incidence in Five Continents, Vol. X (CI5X) and "GLOBOCAN 2012 v1.0, cancer incidence and mortality worldwide: IARC CancerBase No. 11" [9, 10], Colquhoun et al. estimated 260,000 cases of GCC worldwide in 2012 , comprising $27 \%$ of the total gastric cancer cases, most of which occurred in Eastern/Southeastern Asia (59\%), followed by Central Asia (15\%). More than half of the total cases occurred in China (135,000; 52\%) [2]. In high-risk areas in Asia [11-13], the incidence of GCC shows a characteristic geographic aggregation with esophageal cancer (EC). China has six high-risk EC regions, including the Taihang Mountain area, the Qingling Mountain area, North of Sichuan Province, the Dabie Mountain area, East of Guangdong Province, and the Subei area [5]. The Chaoshan high-risk area east of Guangdong is the only littoral high-risk area. Our previous epidemiological study showed an extremely high incidence of EC (74.47/100,000) and GCC (34.81/ 100,000) on Nan'ao Island in the Chaoshan area from 1995 to 2004 [11].

EC and GCC used to be treated as a single disease because of the coincidence of the two cancers, similar clinical symptoms, and limited techniques to distinguish them. Since the 1990s, with widespread use of endoscopy and a new classification by the World Health Organization (WHO), GCC is diagnosed as a disease different from EC in high-risk areas in China; however, the tumorigenesis, pathogenesis, development, and prognosis of GCC from high-risk areas are poorly understood. Although GCC has the same geographic distribution as EC in China [14], previous study showed that they differ in histopathology, although this was not analyzed statistically [15]. Recent epidemiological data have shown that the incidence of EC is declining as compared with an increasing trend of GCC in high-risk areas [16]. GCC in high-risk areas is also different from carcinomas of the GEJ studied primarily in Caucasian populations, which are believed to be related to gastroesophageal reflux disease (GERD) [3] and Barrett's esophagus (BE) $[17,18]$. BE-related GCC are rare in China [19-21]. GERD prevalence in China was reported in some areas of China, and it is reported GERD rates in Hong Kong, China, have risen over the last decade. However the incidence rate of GERD in China is still much lower than that reported in Western countries [22-24].

Helicobacter pylori (H. pylori) infection has been confirmed as an important factor for distal gastric cancer [25]. Its relationship with GCC remains controversial [26-29]. Our previous study suggested that persistent $H$. pylori infection and the related chronic inflammation may contribute to the high incidence of GCC in the Chaoshan high-risk area [29]. Toll-like receptors are essential for $H$. pylori recognition and they initiate inflammatory pathways that may acquire oncogenic potential [30-32]. Toll-like receptor 4 (TLR4) can recognize lipopolysaccharide, a component of the bacterial cell wall [33]. The relationship between TLR4 and gastric cancer has been well studied [34-38]. However, most of these studies did not distinguish GCC from NGCC. No study has addressed TLR4 protein expression restricted to gastric cardia subtype.
In the present study, we compared the clinicopathologic feature of GCC and EC in the Chaoshan high-risk area in China. TLR4 expression was evaluated by immunohistochemistry in normal gastric cardia mucosa, chronic gastric cardia inflammation, and GCC, to better understand the potential role of TLR4 in gastric cardia carcinogenesis. Despite different classifications and definitions of cancers originating around the GEJ $[39,40]$, we defined GCC as carcinoma in which the epicentre is $\leq 2 \mathrm{~cm}$ below the GEJ, the most accepted definition in China [16].

\section{Results}

2.1. Clinicopathological Results. We included 84 patients with EC (male: female ratio $3: 1$ and mean age $57.14 \pm 10.28$ years) and 99 with GCC (male:female ratio 5.6:1 and mean age $62.7 \pm 7.79$ years). Patients with EC were younger than those with GCC. Although more GCC than EC patients were male, the difference in the male:female ratio between the two groups was not considerable (Table 1).

The median tumor size was larger with GCC than that with EC $(5.92 \pm 2.22$ versus $4.98 \pm 1.51 \mathrm{~cm} ; P=0.001)$ (Table 1). For 66 EC tumors (78.57\%), the epicentres were in the middle thoracic part of the esophagus (Table 1). Representative gross images of EC and GCC are in Figures 1 and 2. The microscopic features of the tumors are summarized in Table 2. Compared with EC tumors, GCC tumors were significantly more poorly differentiated $(P<0.001)$ and exhibited a wider histopathological spectrum. All 84 EC tumors (100\%) were squamous cell carcinoma. In contrast, $75.8 \%$ of GCC tumors were tubular adenocarcinoma; the remainder were mucinous carcinoma (16.2\%), adenosquamous carcinoma (4\%), small-cell undifferentiated carcinoma $(n=2,2 \%)$, squamous cell carcinoma $(n=1,1 \%)$, or a tumor of neuroendocrine phenotype $(n=1,1 \%)$. Representative histology images are in Figures 1 and 2.

A significantly higher proportion of patients with GCC than EC showed advanced-stage disease $(P<0.001)$. In addition, a higher proportion of patients with GCC showed lymph node metastasis and deep infiltration $(P=0.002$ and $P=0.009$, respectively, Table 2 ). The tumor tissues from 25/44 patients with EC (56.81\%) and 49/83 with GCC (59.03\%) were positive for $H$. pylori cytotoxin-associated gene A (CagA), a virulence factor that may damage the gastric mucosa and cause inflammation and cell death. Almost all of the East Asian H. pylori strains are CagA positive. Detecting CagA can be used to detect CagA-positive H.pylori infection [41]. We found no significant difference in the rate of $H$. pylori infection between EC and GCC groups $(P=0.59)$ (Table 3).

2.2. Patient Survival. Survival did not differ among patients who received surgery alone and both surgery and adjuvant therapy, so we chose all 84 EC patients and 99 GCC patients who underwent surgery for survival analysis. The mean survival was shorter with EC than that with GCC (32.14 months, 95\% CI 26.796-37.477 versus 43.05 months, 95\% CI 34.933$51.165)$ but not significantly $(P=0.731)$ (Figure $3(a))$. The cumulative survival with EC was better without than with 
TABLE 1: Clinical and gross features of tumor patients with esophageal cancer (EC) and gastric cardia cancer (GCC).

\begin{tabular}{lccc}
\hline Features & EC & GCC & \multirow{2}{*}{$\begin{array}{l}n=99 \\
\end{array}$} \\
\hline Male : female ratio & $63: 21$ & $84: 15$ & 0.095 \\
Age, years, mean \pm SD & $57.14 \pm 10.28$ & $62.7 \pm 7.79$ & 0.003 \\
Size, cm, mean \pm SD & $4.98 \pm 1.51$ & $5.92 \pm 2.22$ & 0.001 \\
Epicentre location, $n(\%)$ & & & \\
$\quad$ Upper thoracic part & $9(10.71)$ & - & \\
Middle thoracic part & $66(78.57)$ & - & \\
Lower thoracic part & $9(10.71)$ & - & \\
Gastric cardia & - & 99 & \\
\hline
\end{tabular}

lymph node metastasis $(38.83 \pm 4$ versus $25.45 \pm 3.4, P=$ $0.029)$ (Figure 3(b)). The cumulative survival of patients with GCC was better with higher than lower TNM stage (76.4 \pm 20.27 and $67.7 \pm 15.28$ versus $37.86 \pm 4.09, P=0.041$; Figure $3(\mathrm{c})$ ) and without than with lymph node metastasis (65.67 \pm 8.75 versus $34.19 \pm 4.17, P=0.001$; Figure $3(\mathrm{~d})$ ). On multivariable analysis, lymph node metastasis was independently associated with survival with GCC (HR 2.02, 95\% confidence interval [CI] 1.058 to $3.837, P=0.033$ ).

The 3-year survival rate was better for patients with EC than that with GCC ( $40.5 \%$ versus $34.3 \%$ ), but the 5 -year survival rate was poorer with EC than that with GCC $(22.6 \%$ versus $25.3 \%)$. Neither of these differences was statistically significant $(P=0.392$ and $P=0.678$, resp.).

2.3. TLR4 Expression in Gastric Cardia Specimens. In Table 4 and Figures 4 and 5, TLR4 expression in gastric cardia tissue is shown. Among the 98 nonmalignant gastric cardia tissues examined, 11 normal epithelia without inflammation had the lowest score for TLR4 expression, and TLR4 was not detectable in 5 of them. On immunohistochemistry, TLR4 expression was higher in inflamed epithelia than that in gastric cardia mucosa (Figure 4) but did not differ between mild and severe inflammation. TLR4 expression was detectable in most of the 97/103 GCC cases (94.17\%). Moreover, strong TLR4 staining was found in well- and moderately differentiated GCC cases with tubular structures but weak or negative TLR4 staining in poorly differentiated tumors (Figure 5). In mucosa with inflammation, TLR4 was expressed in a polarized manner, particularly at the basolateral membrane. In contrast, cancer cells expressed TLR4 diffusely throughout the cytoplasm even in the nucleus. We found a statistically significant trend for increasing TLR4 expression from normal mucosa to gastric cardia inflammation and carcinoma $(P<0.05)$ (Table 4$)$.

\section{Discussion}

In the present study, we compared clinical-pathological features between EC and GCC and described for the first time TLR4 expression restricted to the gastric cardia epithelium. Patients with GCC were significantly older and had a higher male:female ratio than patients with EC, although the difference in the latter variable was not considerable. $92.92 \%(92 / 99)$ of GCC patients were older than 50 years, and $72.62 \%(61 / 84)$ of EC patients were older than 50 years, indicating middle age and elderly people are high-risk group for both GCC and EC, especially for GCC. The mean age for GCC increased compared to the mean age of patients in the 1980s and 1990s [42]. It might be related to the Chinese social aging. A male predominance in the incidence of GCC and EC has been reported worldwide [11, 42, 43], and the male predominance is weaker in EC than that in GCC in this study. An assessment in China high-risk area for GCC indicated male:female ratio ranged from 1.68 to $5.6[15,16]$. The reason for male predominance in GCC is still unclear. Although tobacco smoking is more prevalent in men than in women in China, the male predominance of GCC is unlikely to relate to this factor. A cohort study following 2 million person-years at risk indicated that the male predominance in GCC was similar among smokers and nonsmokers [44]. In China high-risk area, the male predominance in GCC may be caused by some sex-related genetic factors which need to be further studied.

GCC tumors were, on average, larger and more poorly differentiated, were of higher pathological stage, and were more likely to have lymph node metastasis and deeper invasion than EC tumors. Patients with GCC were more likely to have $H$. pylori infection, although the difference did not reach statistical significance. The GCC group had more histological variants than the EC group. GCC included adenocarcinoma, squamous cell carcinoma, adenosquamous carcinoma, smallcell undifferentiated carcinoma, and neuroendocrine carcinoma. In contrast, in ECs, all tumors were squamous epithelial cell carcinoma. The most common location for EC in our sample was the middle thoracic part of the esophagus, whereas in the Western countries, EC originates mainly from the lower part of the esophagus, and the most common histological type is adenocarcinoma $[17,45]$. Survival was poorer for both EC and GCC patients with than without lymph node metastasis. We found similar overall survival among our EC and GCC patients, even though the latter had a significantly higher proportion of lymph node metastasis and stage 3 and 4 tumors at the time of diagnosis. In light of these observations, GCC in high-risk areas in China has clinical and pathological features that differ from those of EC from the same area.

Although different studies have shown some shared genetic risk factors between EC and GCC from high-risk areas in China [14, 46-48], the different histology and surrounding anatomical structure of the esophagus and gastric cardia and respective risk factors indicate differences between EC and GCC [49].

Huang et al. performed a study of the clinical and pathological features of GEJ carcinomas in Chinese and US patients. In terms of their data from the United States, patients with GCC in our group showed larger tumor size $(5.92 \pm 2.22 \mathrm{~cm}$ versus $3.5 \pm 2.2 \mathrm{~cm})$, lower 3 -year ( $34.3 \%$ versus $43 \%)$ and 5 -year (25.3\% versus $28 \%$ ) survival, and higher disease stage than the US patients [45]. Compared with adenocarcinoma of the GEJ in patients with BE mucosa from America, our patients with GCC showed deeper invasion, 


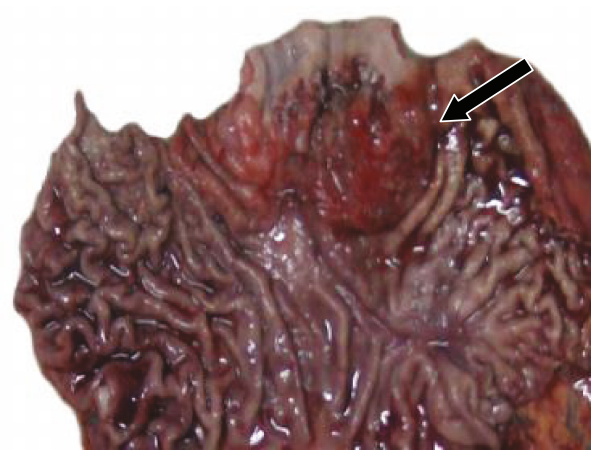

(a)

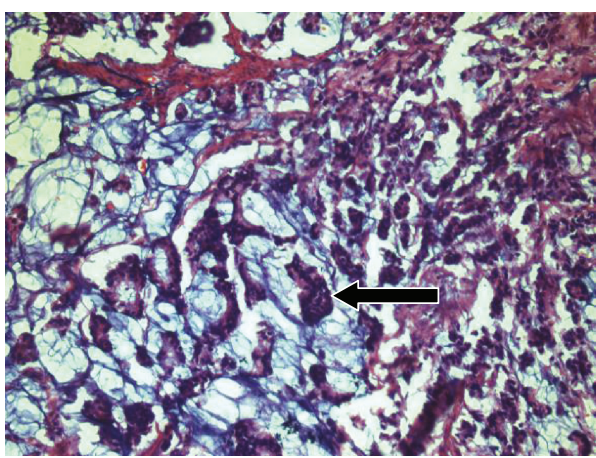

(c)

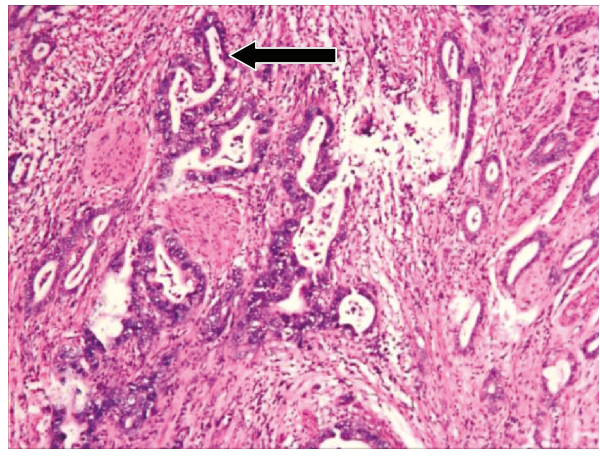

(b)

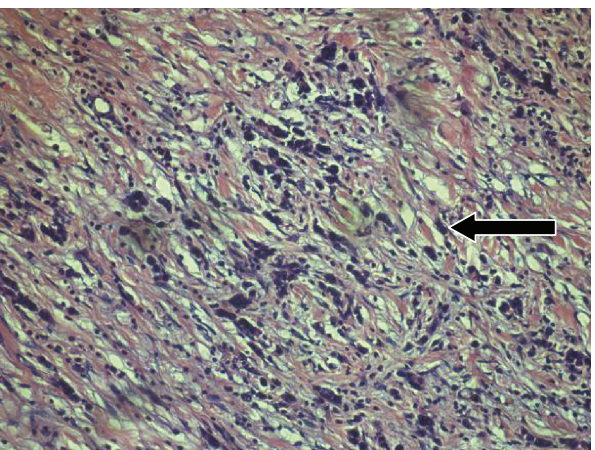

(d)

FIGURE 1: Representative images of gastric cardia cancer (GCC). (a) Gross image of GCC below the gastroesophageal junction (arrow). (b) Histology of GCC with tubular formation (arrow). (c) Histology of mucinous GCC, arrow indicating tumor cells. (d) Histology of small-cell undifferentiated carcinoma, arrow indicating tumor cells.

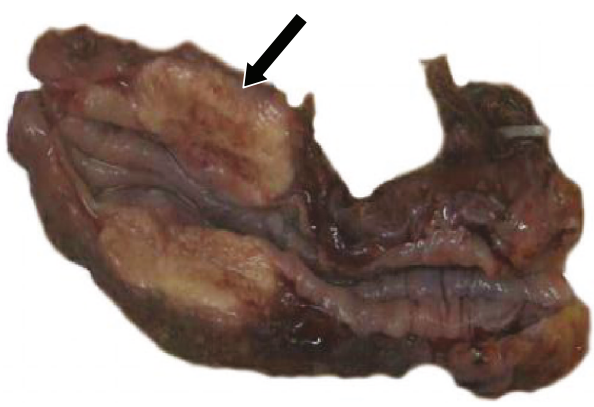

(a)

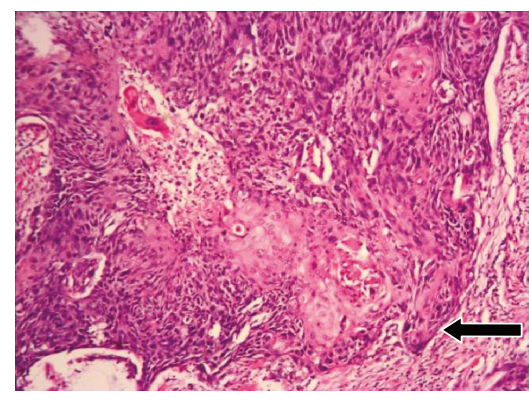

(b)

FIGURE 2: Representative images of esophageal cancer (EC). (a) Gross image of EC (arrow). (b) Histology of squamous cell EC, arrow indicating cancer nest.

higher disease stage, more lymph node metastases, and lower 5 -year survival [50]. When comparing data on adenocarcinoma of the GEJ in patients from Japan, with the center located between $1 \mathrm{~cm}$ above and $2 \mathrm{~cm}$ below the GEJ [51], for our patients, the depth of tumor invasion was deeper, nodal metastases were more frequent, and the differentiation and 5-year survival were worse. In a meta-analysis, the 5-year survival for patients with cancer of the gastric cardia varied from $35 \%$ to $54.6 \%$ in China $[52,53]$. All of these studies reported higher survival rates than we found in our study. Although these studies had discrepancies and overlapping descriptions of tumor location, careful consideration of these results suggests that GCC in the Chaoshan high-risk area in China is more aggressive with a worse prognosis.

Adenocarcinoma of the distal esophagus and Barrett's esophagus-related diseases remain uncommon in China. A large-scale longitudinal clinical and histological data was analyzed on 5401 esophageal cancer (EC) patients diagnosed during 10-year period (2002-2011) at Henan Taihang Mountain high risk area in China. All 217 esophageal adenocarcinoma (EAC) patients from these 5401 EC patients were examined, and EAC was relatively rare and accounted for approximately $5 \%$ of all esophageal cancers. Only 10 out of $217(4.6 \%)$ EAC cases were detected to have any evidence 
TABLE 2: Comparison of histopathology and pathological staging.

\begin{tabular}{|c|c|c|c|}
\hline Microscopic features & $\begin{array}{c}\text { EC } \\
n=84\end{array}$ & $\begin{array}{c}\text { GCC } \\
n=99\end{array}$ & $P$ \\
\hline Tumor differentiation & & & $<0.001$ \\
\hline Well & $29(34.5)$ & $5(5.1)$ & \\
\hline Moderate & $50(59.5)$ & $49(49.5)$ & \\
\hline Poor & $5(6.0)$ & $45(45.5)$ & \\
\hline \multicolumn{4}{|l|}{ Histology type } \\
\hline Tubular adenocarcinoma & $0(0)$ & $75(75.8)$ & $<0.001$ \\
\hline Mucinous carcinoma & $0(0)$ & $16(16.2)$ & \\
\hline $\begin{array}{l}\text { Small-cell undifferentiated } \\
\text { carcinoma }\end{array}$ & $0(0)$ & $2(2.0)$ & \\
\hline Squamous cell carcinoma & $84(100)$ & $1(1.0)$ & \\
\hline Adenosquamous & $0(0)$ & $4(4.0)$ & \\
\hline Neuroendocrine carcinoma & $0(0)$ & $1(1.0)$ & \\
\hline Lymph node metastasis & $42(50)$ & $72(72.7)$ & 0.002 \\
\hline Serosal invasion & $67(70.8)$ & $92(92.9)$ & 0.009 \\
\hline \multicolumn{4}{|l|}{ TNM stage } \\
\hline 0 & $0(0)$ & $0(0)$ & $<0.001$ \\
\hline 1 & $0(0)$ & $5(5.1)$ & \\
\hline 2 & $\begin{array}{c}40 \\
(47.62)\end{array}$ & $10(10.1)$ & \\
\hline 3 & $42(50)$ & $84(84.8)$ & \\
\hline 4 & $2(2.4)$ & $0(0)$ & \\
\hline
\end{tabular}

Data are $n(\%)$.

TABle 3: Helicobacter pylori infection.

\begin{tabular}{lccc}
\hline H. pylori infection & EC & GCC & P \\
& $n=44$ & $n=83$ & $P$ \\
\hline Positive & $19(43.18)$ & $40(48.19)$ & 0.59 \\
Negative & $25(56.81)$ & $43(51.81)$ & \\
\hline
\end{tabular}

Data are $n(\%)$.

of Barrett's esophagus [20]. Though the GERD prevalence in GCC high-risk area in China is unclear, the prevalence of GERD symptoms in South China has varied from 2.3\% to $3.8 \%$, much lower than that in the Western countries $[22,23]$. Patients with GCC in China and those in the Western countries might have different genetic polymorphisms, lifestyles, diet, and environmental influences [45]. H. pylori infection is the primary risk factor for distal gastric carcinomas in the Chinese population [54]. A case-cohort study with long-term follow-up in the Linzhou high-risk area found a strong association of GCC with $H$. pylori infection [55]. Our previous study found that $H$. pylori infection may contribute to the high incidence of GCC and esophageal squamous cell carcinoma in the Chaoshan region $[29,56]$. Gastric carcinomas develop on the background of chronic active $H$. pylori gastritis via the epithelial precursor lesions. It is believed that a virulent bacterium in a genetically susceptible host is associated with more severe chronic inflammation, and this longterm inflammation may lead to cancer [31, 57]. Observing the adjacent tissue of GCC, we found that most cases showed chronic inflammatory cell infiltration. We speculated the carcinogenesis of GCC in high-risk area might be related to chronic inflammation similar to the NGCC.

Previous studies suggested that TLR4 expression might be the link between $H$. pylori infection and cancer $[31,37,58]$, and this expression pattern is not significantly changed after the eradication of bacteria [59]. In the present study, TLR4 expression was evaluated in a cohort of gastric cardia tissues. To our knowledge, our study is the first to evaluate TLR4 protein expression from normal mucosa to different degree of inflammation and carcinoma restricted to gastric cardia tissue. TLR4 expression gradually increased from normal mucosa to gastric cardia inflammation and carcinoma, thereby providing pathological evidence that TLR4 expression is involved in GCC inflammation and carcinogenesis. Similar to other studies, our study showed that normal gastric cardia cancer has a very low expression of TLR4 $[58,60]$. TLR4 expression was greatly increased during chronic inflammation, and there was no significant difference between mild inflammation and severe inflammation, suggesting changes in innate immune activation between normal and mild inflammation. As we have seen, GCC has the highest and diffuse TLR4 expression. TLR4 expression was not only in the cytoplasm but also in the nucleus. It was speculated that at this phase, the presence of infection was not absolutely necessary for epithelial stimulation [58]. Though we did not detect TLR4 expression in EC tissue, previous studies showed TLR4 appeared important to the pathogenesis of esophageal squamous cell carcinoma [61]. Confirming the potential role of TLR4 in the progression of gastric lesions, some studies associated TLR4 polymorphisms with the risk of gastric cancer [34, 62-64]. TLR4+896A $>\mathrm{G}$ polymorphism was reported as a risk factor for NGCC and its precursors. In contrast, prevalence of TLR4+896G was not significantly increased in GCC [34]. Considering that GCC has different characteristics from NGCC, more molecular and functional studies about TLR4 in GCC are necessary, and distinguishing GCC from NGCC is encouraged.

In summary, this study showed that GCC carcinomas are biologically different from EC carcinomas in the Chaoshan high-risk area in China, although they share genetic risk factors and similar geographic aggregation. GCC in this high-risk area displays different characteristics from those of GEJ carcinomas in developed countries as well. We detected TLR4 expression in gastric cardia epithelial cells and demonstrate a progressive increase in TLR4 expression from normal gastric cardia tissue, gastric cardia inflammation, and GCC, which suggests that TLR4 plays a role in GCC carcinogenesis.

\section{Methods}

4.1. Study Group. All surgical pathology reports with a final diagnosis of EC and GCC were collected from the Tumor Hospital and the First Affiliated Hospital of Shantou University Medical College in China. Not all patients underwent radiotherapy and chemotreatment postoperatively. All cases were divided into EC and GCC groups based on the location of the tumor epicentre. Inclusion criteria were (1) surgical 


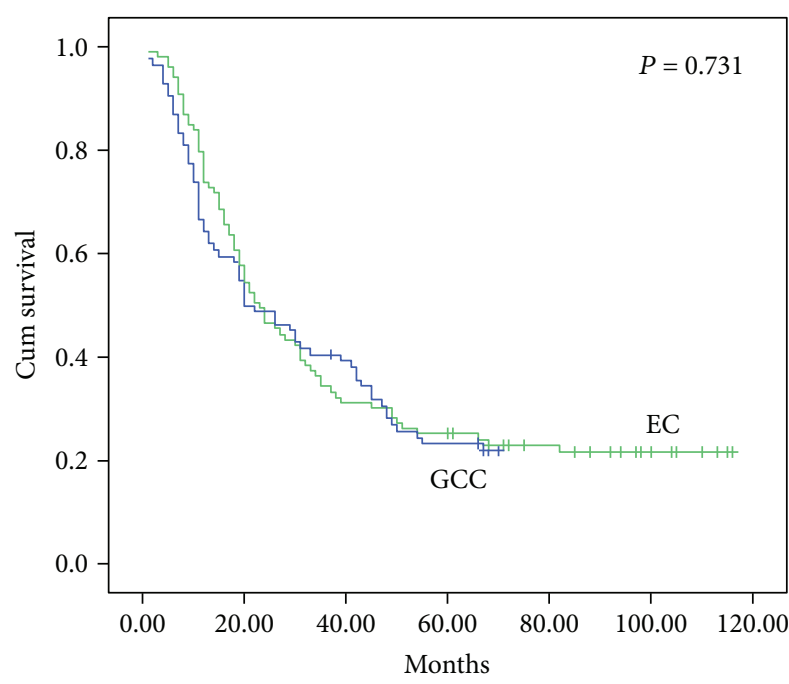

(a)

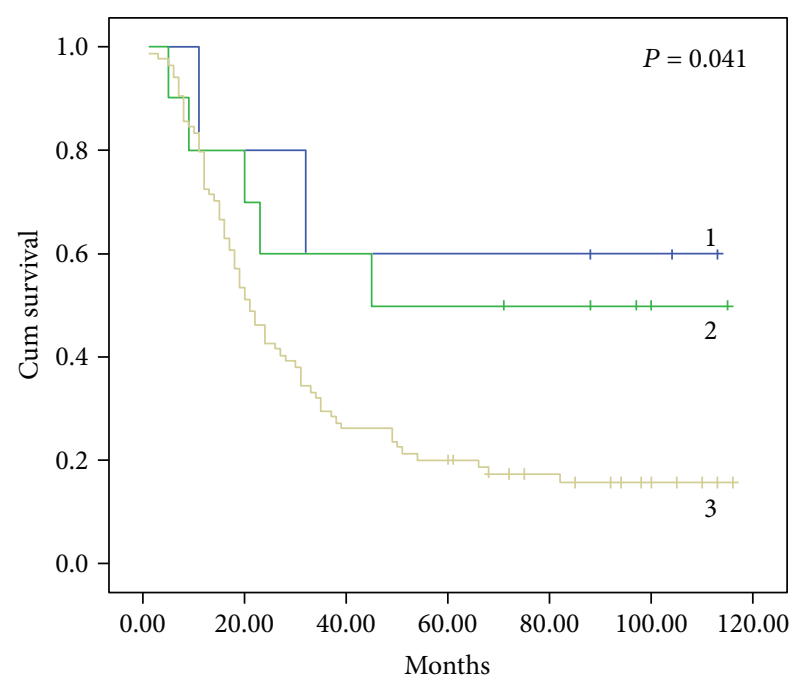

(c)

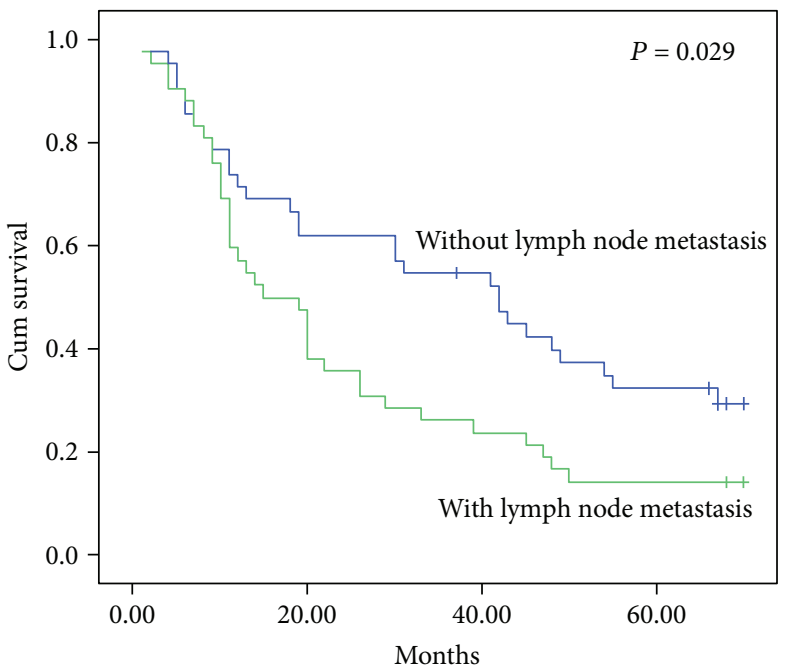

(b)

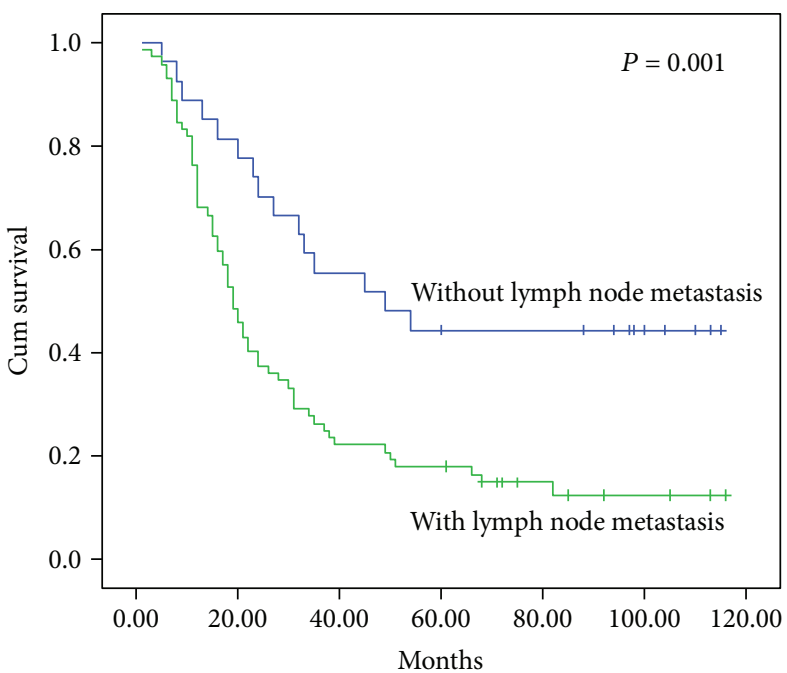

(d)

FIGURE 3: Kaplan-Meier survival curves for patients with GCC and EC. (a) Overall survival. (b) Cumulative survival with EC with and without lymph node metastasis. (c) Cumulative survival with GCC by TNM stage. 1: stage 1, 2: stage 2, 3: stage 3. (d) Cumulative survival with GCC with and without lymph node metastasis.

TABLE 4: Immunohistochemical evaluation of TLR4 expression.

\begin{tabular}{lcccc}
\hline & Normal & Mild carditis & Severe carditis & Carcinoma \\
$n=11$ & $n=44$ & $n=43$ & $n=103$ \\
\hline TLR4 & $12.72 \pm 15.71$ & $28.63 \pm 21.65^{*}$ & $25.58 \pm 22.23^{*}$ & $63.67 \pm 39.61^{* \#}$ \\
\hline
\end{tabular}

TLR4: Toll-like receptor 4 . Data are mean $\pm \mathrm{SD} ;{ }^{*} P<0.05$ versus normal mucosa; ${ }^{\#} P<0.05$ versus mild or severe inflammation.

resection of tumors with lymph node dissection and (2) for GCC, the center of cancer within $2 \mathrm{~cm}$ of the GEJ on the gastric side. The GEJ was as defined by the WHO [46]. Most of the GCC tumors belonged to the AEG type II according to the Siewert classification [65]. Surgical details were collected principally from surgical notes and pathology findings of the resection specimen. GCCs were staged by the gastric TNM system, and ECs were staged by the esophageal TNM system, according to the American Joint Committee on Cancer
Staging Manual [66]. Patients were followed for survival status by telephone or personal interview with the patient or family members. Patient consent for surgery and follow-up visit was obtained in all cases before surgical resection was performed. Informed consent was obtained from all patients. The methods were carried out in accordance with the approved guidelines. The Medical Ethics Committee of Shantou University Medical College approved the study protocol. 


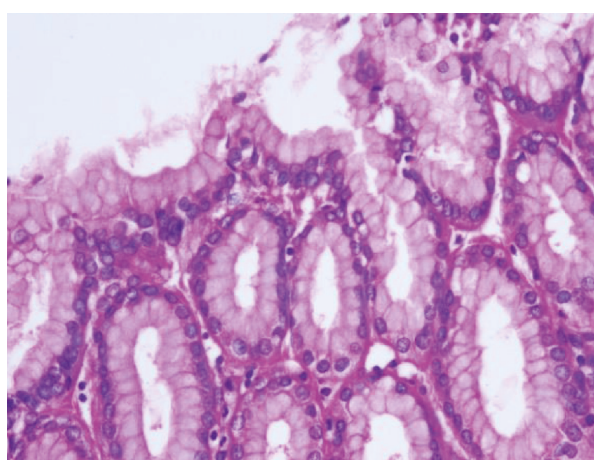

(a)

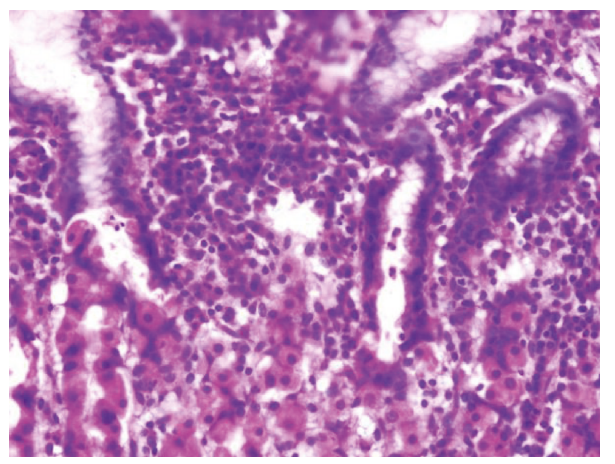

(c)

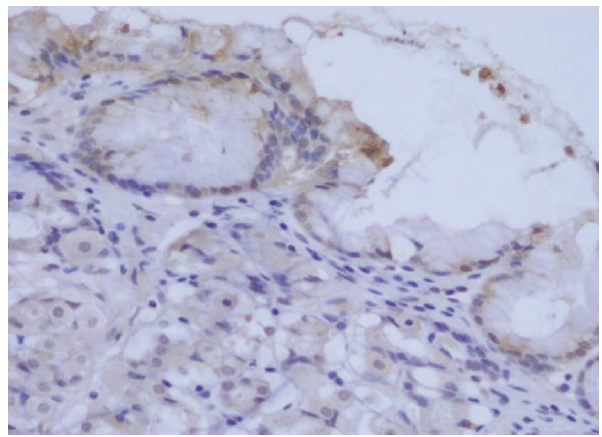

(e)

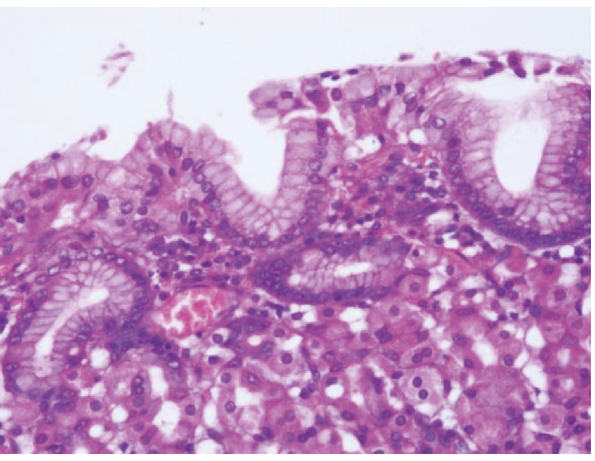

(b)

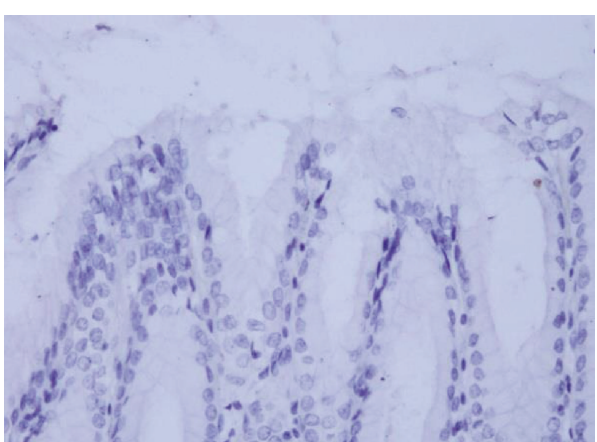

(d)

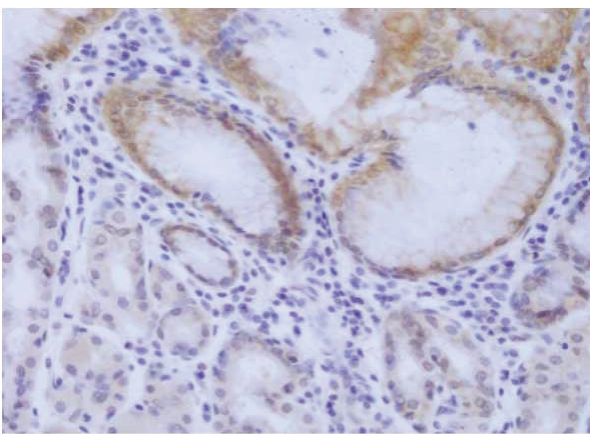

(f)

FIGURE 4: Representative IHC staining for TLR4 in gastric cardia mucosae with different degrees of chronic inflammation: (a) normal, (b) mild inflammation, (c) severe inflammation, (d) no immunostaining in normal mucosae, (e) weak positive staining in mucosae with mild inflammation, and (f) moderate positive staining in mucosae with severe inflammation.

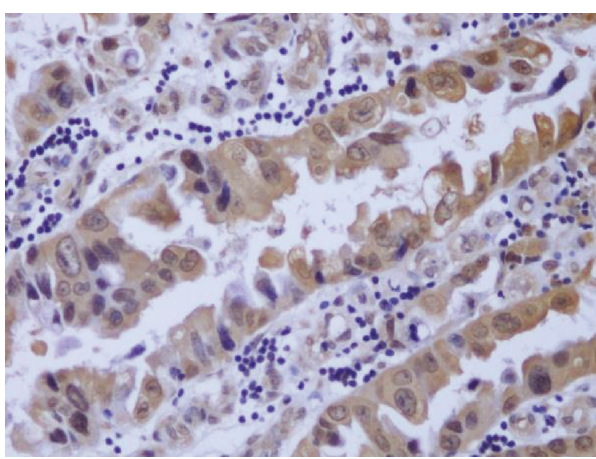

(a)

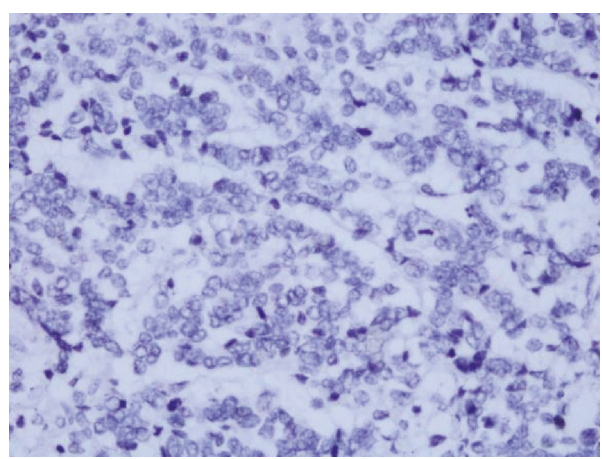

(b)

FIGURE 5: IHC staining for TLR4 in GCC tissue. (a) Strong positive staining in well-differentiated GCC cases with tubular structure. (b) Negative TLR4 staining in poorly differentiated tumor. 
4.2. DNA Extraction and Amplification for Detecting H. pylori Infection. A total of 127 gastrointestinal mucosal tissue samples were collected from 83 patients with GCC and 44 with EC in the Tumor Hospital of Shantou University Medical College in China. Tissue DNA was extracted using a commercially available kit (Pure Link Genomic DNA Mini Kit, Invitrogen) according to the manufacturer's instructions [67]. The primers used were derived from the internal $300 \mathrm{bp}$ fragment of the CagA as described [68]. The primer sequences used to detect CagA were $5^{\prime}$-ACCCTAGTCGG TAA TGGG- $3^{\prime}$ and $5^{\prime}$-GCA AT TT TGT TAATCCGG TC- $3^{\prime}$. These yielded a DNA fragment of 300 base pairs. A reaction mixture contained $3 \mu \mathrm{L}$ extracted DNA, $4 \mu \mathrm{L}$ primer, $3 \mu \mathrm{L}$ of $10 x$ PCR buffer, $0.3 \mu \mathrm{L}$ AmpliTaq DNA polymerase, and $3 \mu \mathrm{L} \mathrm{dNTP}$. The amplification cycle consisted of an initial denaturation at $94^{\circ} \mathrm{C}$ for $3 \mathrm{~min}$, followed by 34 cycles at $94^{\circ} \mathrm{C}$ for $30 \mathrm{~s}, 50^{\circ} \mathrm{C}$ for $45 \mathrm{~s}, 72^{\circ} \mathrm{C}$ for $1 \mathrm{~min}$, and a final extension at $72^{\circ} \mathrm{C}$ for $10 \mathrm{~min}$. PCR products were analyzed by electrophoresis on a $2 \%$ agarose gel stained with ethidium bromide.

4.3. Immunohistochemistry. Immunohistochemical (IHC) staining involved the Envision Labeled Peroxidase System (Dako, Carpinteria, CA). Paraffin-embedded samples were sectioned at $4 \mu \mathrm{m}$. Each sample was deparaffinized in xylene, rehydrated in a graded ethanol series, then preincubated with $3 \%$ hydrogen peroxide for $10 \mathrm{~min}$. Antigen retrieval was performed by microwave heating. Following incubation in $10 \mathrm{mmol} / \mathrm{L}$ citrate buffer for $20 \mathrm{~min}$, sections were incubated with primary antibody for TLR4 (rabbit, 1:100, Proteintech) at $4^{\circ} \mathrm{C}$ overnight, then horseradish peroxidase-conjugated goat antirabbit IgG antibody at $37^{\circ} \mathrm{C}$ for $30 \mathrm{~min}$, and counterstained with haematoxylin. Images were captured under a Leica IM50 microscope (Imagic Bildverarbeitung AG, Wetzlar, Germany).

IHC slides were evaluated by two experienced pathologists in a blinded manner. Staining intensity score (0-3) was considered according to a subjective evaluation of the intensity of marked cells (0: no immunostaining; 1 : weak positive staining; 2 : moderate positive staining; 3 : strong positive staining). The overall staining intensity $(0-3)$ was multiplied by the proportion of positive cells $(0-100 \%)$, and all values were added to generate a final score ranging from 0 to 300 [69].

4.4. Chronic Inflammation Grading. Inflammation in the gastric cardia tissue was graded as normal, mild, and severe according to the updated Sydney System [70]. The normal gastric mucosa contains only individual $(0-5)$ scattered inflammatory cells in the lamina propria. Mild inflammation contains 5 to 30 inflammatory cells in the lamina propria per high-power $(\times 40$ objective) microscopic field or between the foveolae. More than 30 inflammatory cells per high-power field was considered severe inflammation.

4.5. Statistical Analysis. Differences in frequencies between the two groups were analyzed by using chi-square or Fisher's exact test, as appropriate. Survival was estimated by the Kaplan-Meier method with a log-rank test. Cox regression analysis was used to identify risk factors for overall survival. $t$-test for trend was used to evaluate the trend in increase or decrease in expression. All statistical analyses involved the use of SPSS v16 (SPSS, Chicago, IL, USA). $P<0.05$ was considered statistically significant.

\section{Conflicts of Interest}

The authors declare no conflicts of interest.

\section{Acknowledgments}

This work was supported by the Department of Education, Guangdong Government under the Top-tier University Development Scheme for Research and Control of Infectious Diseases, the Natural Science Foundation of China (Grant no. 81572684), the Natural Science Foundation of Guangdong Province (Grant no. 2014A030310139), and the National Natural Science Foundation of China (Grant no. 81702717).

\section{References}

[1] D. M. Parkin, S. L. Whelan, J. Ferlay, L. Teppo, and B. D. Thomas, Cancer Incidence In Five Continents, IARC Sci Publ No. 155. IARC, Lyon, 2002.

[2] A. Colquhoun, M. Arnold, J. Ferlay, K. J. Goodman, D. Forman, and I. Soerjomataram, "Global patterns of cardia and non-cardia gastric cancer incidence in 2012," Gut, vol. 64, no. 12, pp. 1881-1888, 2015.

[3] S. J. Crane, Richard Locke G3rd, W. S. Harmsen et al., "The changing incidence of oesophageal and gastric adenocarcinoma by anatomic sub-site," Alimentary Pharmacology and Therapeutics, vol. 25, no. 4, pp. 447-453, 2007.

[4] J. Shi, Q. Sun, B. Y. Xu et al., "Changing trends in the proportions of small $(\leq 2 \mathrm{~cm})$ proximal and non-proximal gastric carcinomas treated at a high-volume tertiary medical center in China," Journal of Digestive Diseases, vol. 15, no. 7, pp. 359-366, 2014.

[5] Z. C. Z. DONG, "Incidence and geographic characteristics of esophagus-gastric junction adenocarcinoma," Chinese Journal of Clinical Oncology, vol. 38, no. 1, pp. 57-60, 2011.

[6] F. Carneiro and P. Chaves, "Pathologic risk factors of adenocarcinoma of the gastric cardia and gastroesophageal junction," Surgical Oncology Clinics of North America, vol. 15, no. 4, pp. 697-714, 2006.

[7] R. D. Odze, "Pathology of the gastroesophageal junction," Seminars in Diagnostic Pathology, vol. 22, no. 4, pp. 256-265, 2005.

[8] J. Olsen, M. L. M. Espersen, P. Jess, L. T. Kirkeby, and J. T. Troelsen, "The clinical perspectives of CDX2 expression in colorectal cancer: a qualitative systematic review," Surgical Oncology, vol. 23, no. 3, pp. 167-176, 2014.

[9] D. Forman, F. Bray, D. H. Brewster et al., Cancer Incidence in Five Continents, Vol. X (Electronic Version)July 2014, http://ci5.iarc.fr.

[10] J. Ferlay, I. Soerjomataram, M. Ervik et al., "GLOBOCAN 2012 v1.0, cancer incidence and mortality worldwide: IARC CancerBase No. 11," July 2014, http://globocan.iarc.fr.

[11] M. Su, M. Liu, D. P. Tian et al., “Temporal trends of esophageal cancer during 1995-2004 in Nanao Island, an extremely high- 
risk area in China," European Journal of Epidemiology, vol. 22, no. 1, pp. 43-48, 2007.

[12] M. Babaei, F. Pourfarzi, A. Yazdanbod et al., "Gastric cancer in Ardabil, Iran - a review and update on cancer registry data," Asian Pacific Journal of Cancer Prevention, vol. 11, no. 3, pp. 595-599, 2010.

[13] N. Taghavi, D. Nasrollahzadeh, S. Merat et al., "Epidemiology of upper gastrointestinal cancers in Iran: a sub site analysis of 761 cases," World Journal of Gastroenterology, vol. 13, no. 40, pp. 5367-5370, 2007.

[14] L. D. Wang, F. Y. Zhou, X. M. Li et al., "Genome-wide association study of esophageal squamous cell carcinoma in Chinese subjects identifies a susceptibility locus at PLCE1," Nature Genetics, vol. 42, no. 9, pp. 759-763, 2010.

[15] C. L. Fu, M. Su, and D. P. Tian, "Clinipathologic analyses of 16954 cases with malignant tumor at esophageal and oesophagogastric junction in Chaoshan area, Guangdong Province," China Cancer, vol. 17, no. 5, pp. 421-425, 2008.

[16] C. W. Peng Xiabiao, C. Zhifeng, L. Zhiheng, O. Zhixiong, and W. Kuangrong, "Epidemiology of gastric cardia cancer in China," Chinese Archives of General Surgery, vol. 8, no. 2, pp. 156-159, 2014.

[17] P. Chandrasoma, K. Wickramasinghe, Y. Ma, and T. DeMeester, "Adenocarcinomas of the distal esophagus and "gastric cardia" are predominantly esophageal carcinomas," The American Journal of Surgical Pathology, vol. 31, no. 4, pp. 569-575, 2007.

[18] B. A. Whitson, S. S. Groth, Z. Li, R. A. Kratzke, and M. A. Maddaus, "Survival of patients with distal esophageal and gastric cardia tumors: a population-based analysis of gastroesophageal junction carcinomas," The Journal of Thoracic and Cardiovascular Surgery, vol. 139, no. 1, pp. 43-48, 2010.

[19] P. H. Tseng, Y. C. Lee, H. M. Chiu et al., "Prevalence and clinical characteristics of Barrett's esophagus in a Chinese general population," Journal of Clinical Gastroenterology, vol. 42, no. 10, pp. 1074-1079, 2008.

[20] S. Liu, J. Y. Dai, L. Yao et al., "Esophageal adenocarcinoma and its rare association with Barrett's esophagus in Henan, China," PLoS One, vol. 9, no. 10, article e110348, 2014.

[21] Q. Huang, D. C. Fang, C. G. Yu, J. Zhang, and M. H. Chen, "Barrett's esophagus-related diseases remain uncommon in China," Journal of Digestive Diseases, vol. 12, no. 6, pp. 420427, 2011.

[22] M. Chen, L. Xiong, H. Chen, A. Xu, L. He, and P. Hu, "Prevalence, risk factors and impact of gastroesophageal reflux disease symptoms: a population-based study in South China," Scandinavian Journal of Gastroenterology, vol. 40, no. 7, pp. 759-767, 2005.

[23] V. P.-Y. Tan, B. C. Y. Wong, W. M. Wong et al., "Gastroesophageal reflux disease: cross-sectional study demonstrating rising prevalence in a Chinese population," Journal of Clinical Gastroenterology, vol. 50, no. 1, pp. e1-e7, 2016.

[24] H. B. El-Serag, "Time trends of gastroesophageal reflux disease: a systematic review," Clinical Gastroenterology and Hepatology, vol. 5, no. 1, pp. 17-26, 2007.

[25] C. de Martel, J. Ferlay, S. Franceschi et al., "Global burden of cancers attributable to infections in 2008: a review and synthetic analysis," The Lancet Oncology, vol. 13, no. 6, pp. 607615, 2012.

[26] Helicobacter and Cancer Collaborative Group, "Gastric cancer and Helicobacter pylori: a combined analysis of 12 case control studies nested within prospective cohorts," Gut, vol. 49, no. 3, pp. 347-353, 2001.

[27] F. Kamangar, S. M. Dawsey, M. J. Blaser et al., "Opposing risks of gastric cardia and noncardia gastric adenocarcinomas associated with Helicobacter pylori seropositivity," Journal of the National Cancer Institute, vol. 98, no. 20, pp. 1445-1452, 2006.

[28] S. J. Cho, I. J. Choi, C. G. Kim et al., "Helicobacter pylori seropositivity is associated with gastric cancer regardless of tumor subtype in Korea," Gut and Liver, vol. 4, no. 4, pp. 466-474, 2010.

[29] Y. Wang, S. Liu, Y. Zhang et al., "Helicobacter pylori infection and gastric cardia cancer in Chaoshan region," Microbes and Infection, vol. 16, no. 10, pp. 840-844, 2014.

[30] S. Akira and K. Takeda, "Toll-like receptor signalling," Nature Reviews Immunology, vol. 4, no. 7, pp. 499-511, 2004.

[31] P. Pimentel-Nunes, N. Gonçalves, I. Boal-Carvalho et al., "Helicobacter pylori induces increased expression of Toll-like receptors and decreased Toll-interacting protein in gastric mucosa that persists throughout gastric carcinogenesis," Helicobacter, vol. 18, no. 1, pp. 22-32, 2013.

[32] N. Castaño-Rodríguez, N. O. Kaakoush, A. L. Pardo, K.-L. Goh, K. M. Fock, and H. M. Mitchell, "Genetic polymorphisms in the toll-like receptor signalling pathway in Helicobacter pylori infection and related gastric cancer," Human Immunology, vol. 75, no. 8, pp. 808-815, 2014.

[33] K. Takeda, T. Kaisho, and S. Akira, "Toll-like receptors," Annual Review of Immunology, vol. 21, no. 1, pp. 335-376, 2003.

[34] G. L. Hold, C. S. Rabkin, W. H. Chow et al., "A functional polymorphism of toll-like receptor 4 gene increases risk of gastric carcinoma and its precursors," Gastroenterology, vol. 132, no. 3, pp. 905-912, 2007.

[35] H. Huhta, O. Helminen, J. H. Kauppila et al., “The expression of Toll-like receptors in normal human and murine gastrointestinal organs and the effect of microbiome and cancer," The Journal of Histochemistry \& Cytochemistry, vol. 64, no. 8, pp. 470-482, 2016.

[36] G. Li, Z. Wang, J. Ye et al., "Uncontrolled inflammation induced by AEG-1 promotes gastric cancer and poor prognosis," Cancer Research, vol. 74, no. 19, pp. 5541-5552, 2014.

[37] X. Yuan, Y. Zhou, W. Wang et al., "Activation of TLR4 signaling promotes gastric cancer progression by inducing mitochondrial ROS production," Cell Death \& Disease, vol. 4, no. 9, article e794, 2013.

[38] B. Schmaußer, M. Andrulis, S. Endrich, H.-K. Müller-Hermelink, and M. Eck, "Toll-like receptors TLR4, TLR5 and TLR9 on gastric carcinoma cells: an implication for interaction with Helicobacter pylori," International Journal of Medical Microbiology, vol. 295, no. 3, pp. 179-185, 2005.

[39] L. Wang Lidong, Z. Yanxia, J. Xinying et al., "Analysis on the occurrence site of gastric cardia adenocarcinoma in high incidence area in Henan," Journal of Zhengzhou University, vol. 42, no. 3, pp. 389-393, 2007.

[40] M. J. Blaser and D. Saito, "Trends in reported adenocarcinomas of the oesophagus and gastric cardia in Japan," European Journal of Gastroenterology \& Hepatology, vol. 14, no. 2, pp. 107113, 2002.

[41] M. Hatakeyama and H. Higashi, "Helicobacter pylori CagA: a new paradigm for bacterial carcinogenesis," Cancer Science, vol. 96, no. 12, pp. 835-843, 2005. 
[42] B. Zheng, Y.-B. Chen, Y. Hu, J.-Y. Wang, Z.-W. Zhou, and J.-H. Fu, "Trend analysis for clinical characteristics and prognosis of adenocarcinoma of cardia," Chinese Journal of Cancer, vol. 29, no. 1, pp. 94-97, 2010.

[43] J. Falk, H. Carstens, L. Lundell, and M. Albertsson, "Incidence of carcinoma of the oesophagus and gastric cardia. Changes over time and geographical differences," Acta Oncologica, vol. 46, no. 8, pp. 1070-1074, 2007.

[44] N. D. Freedman, M. H. Derakhshan, C. C. Abnet, A. Schatzkin, A. R. Hollenbeck, and K. E. L. McColl, "Male predominance of upper gastrointestinal adenocarcinoma cannot be explained by differences in tobacco smoking in men versus women," European Journal of Cancer, vol. 46, no. 13, pp. 2473-2478, 2010.

[45] Q. Huang, X. Fan, A. T. Agoston et al., "Comparison of gastrooesophageal junction carcinomas in Chinese versus American patients," Histopathology, vol. 59, no. 2, pp. 188-197, 2011.

[46] D. Wen, B. Shan, S. Wang et al., "A positive family history of esophageal/gastric cardia cancer with gastric cardia adenocarcinoma is associated with a younger age at onset and more likely with another synchronous esophageal/gastric cardia cancer in a Chinese high-risk area," European Journal of Medical Genetics, vol. 53, no. 5, pp. 250-5, 2010.

[47] C. C. Abnet, N. D. Freedman, N. Hu et al., "A shared susceptibility locus in PLCE1 at 10q23 for gastric adenocarcinoma and esophageal squamous cell carcinoma," Nature Genetics, vol. 42, no. 9, pp. 764-767, 2010.

[48] C. Wu, Z. Hu, Z. He et al., "Genome-wide association study identifies three new susceptibility loci for esophageal squamous-cell carcinoma in Chinese populations," Nature Genetics, vol. 43, no. 7, pp. 679-684, 2011.

[49] S. A. Savage et al., "Variants of the IL8 and IL8RB genes and risk for gastric cardia adenocarcinoma and esophageal squamous cell carcinoma," Cancer Epidemiology, Biomarkers \& Prevention, vol. 13, no. 12, pp. 2251-2257, 2004.

[50] G. Portale, J. H. Peters, J. A. Hagen et al., "Comparison of the clinical and histological characteristics and survival of distal esophageal-gastroesophageal junction adenocarcinoma in patients with and without Barrett mucosa," Archives of Surgery, vol. 140, no. 6, pp. 570-574, 2005.

[51] S. Hasegawa, T. Yoshikawa, H. Cho, A. Tsuburaya, and O. Kobayashi, "Is adenocarcinoma of the esophagogastric junction different between Japan and western countries? The incidence and clinicopathological features at a Japanese highvolume cancer center," World Journal of Surgery, vol. 33, no. 1, pp. 95-103, 2009.

[52] W. L. Fang, C. W. Wu, J. H. Chen et al., "Esophagogastric junction adenocarcinoma according to Siewert classification in Taiwan," Annals of Surgical Oncology, vol. 16, no. 12, pp. 3237-3244, 2009.

[53] S. Y. Law, M. Fok, S. W. Cheng, and J. Wong, "A comparison of outcome after resection for squamous cell carcinomas and adenocarcinomas of the esophagus and cardia," Surgery, Gynecology \& Obstetrics, vol. 175, no. 2, pp. 107-112, 1992.

[54] Q. Huang, J. Shi, Q. Sun et al., "Clinicopathological characterisation of small ( $2 \mathrm{~cm}$ or less) proximal and distal gastric carcinomas in a Chinese population," Pathology, vol. 47, no. 6, pp. 526-532, 2015.

[55] F. Kamangar, Y. L. Qiao, M. J. Blaser et al., "Helicobacter pylori and oesophageal and gastric cancers in a prospective study in China," British Journal of Cancer, vol. 96, no. 1, pp. 172-176, 2007.
[56] W.-s. Li, D.-p. Tian, X.-y. Guan et al., "Esophageal intraepithelial invasion of Helicobacter pylori correlates with atypical hyperplasia," International Journal of Cancer, vol. 134, no. 11, pp. 2626-2632, 2014.

[57] M. R. Amieva and E. M. El-Omar, "Host-bacterial interactions in Helicobacter pylori infection," Gastroenterology, vol. 134, no. 1, pp. 306-323, 2008.

[58] P. Pimentel-Nunes, L. Afonso, P. Lopes et al., "Increased expression of toll-like receptors (TLR) 2, 4 and 5 in gastric dysplasia," Pathology \& Oncology Research, vol. 17, no. 3, pp. 677-683, 2011.

[59] A. C. T. Cadamuro, A. F. T. Rossi, J. M. Biselli-Périco et al., "Effect of Helicobacter pylori eradication on TLR2 and TLR4 expression in patients with gastric lesions," Mediators of Inflammation, vol. 2015, Article ID 481972, 9 pages, 2015.

[60] B. Schmausser, M. Andrulis, S. Endrich et al., "Expression and subcellular distribution of toll-like receptors TLR4, TLR5 and TLR9 on the gastric epithelium in Helicobacter pylori infection," Clinical and Experimental Immunology, vol. 136, no. 3, pp. 521-526, 2004.

[61] I. Sheyhidin, G. Nabi, A. Hasim et al., "Overexpression of TLR3, TLR4, TLR7 and TLR9 in esophageal squamous cell carcinoma," World Journal of Gastroenterology, vol. 17, no. 32, pp. 3745-3751, 2011.

[62] B. R. Achyut, U. C. Ghoshal, N. Moorchung, and B. Mittal, "Association of Toll-like receptor-4 (Asp299Gly and Thr399Ileu) gene polymorphisms with gastritis and precancerous lesions," Human Immunology, vol. 68, no. 11, pp. 901-907, 2007.

[63] A. Hishida, K. Matsuo, Y. Goto et al., "Toll-like receptor 4 +3725 G/C polymorphism, Helicobacter pylori seropositivity, and the risk of gastric atrophy and gastric cancer in Japanese," Helicobacter, vol. 14, no. 1, pp. 47-53, 2009.

[64] T. H. Zou, Z. H. Wang, and J. Y. Fang, "Positive association between Toll-like receptor 4 gene $+896 \mathrm{~A} / \mathrm{G}$ polymorphism and susceptibility to gastric carcinogenesis: a meta-analysis," Tumour Biology, vol. 34, no. 4, pp. 2441-2450, 2013.

[65] J. R. Siewert and H. J. Stein, "Classification of adenocarcinoma of the oesophagogastric junction," British Journal of Surgery, vol. 85, no. 11, pp. 1457-1459, 1998.

[66] American Joint Committee on Cancer, "Esophagus and esophagogastric junction," in AJCC Cancer Staging Manual, pp. 129-144, Springer, New York, NY, USA, 7th edition, 2009.

[67] P. I. Hsu, I.r. Hwang, D. Cittelly et al., "Clinical presentation in relation to diversity within the Helicobacter pylori cag pathogenicity island," The American Journal of Gastroenterology, vol. 97, no. 9, pp. 2231-2238, 2002.

[68] G. D. Liu, L. H. He, Y. X. Gu, N. M. Yang, and J. Z. Zhang, "Using PCR with different primers to detect Helicobacter pylori in gastric biopsy specimens," Journal of Pathogen Biology, vol. 6, no. 6, pp. 401-410, 2011.

[69] Q. Xu, Y. Xu, B. Pan et al., "TTK is a favorable prognostic biomarker for triple-negative breast cancer survival," Oncotarget, vol. 7, no. 49, pp. 81815-81829, 2016.

[70] J. Powell and C. C. McConkey, "Increasing incidence of adenocarcinoma of the gastric cardia and adjacent sites," British Journal of Cancer, vol. 62, no. 3, pp. 440-3, 1990. 


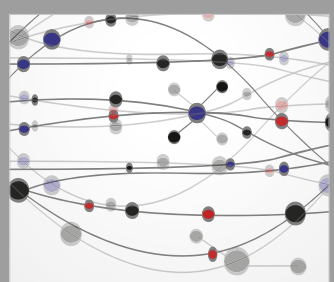

The Scientific World Journal
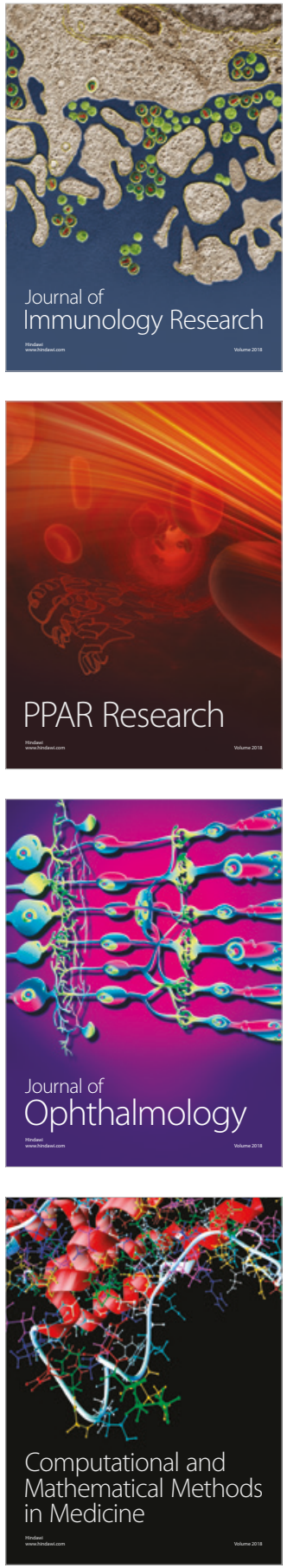

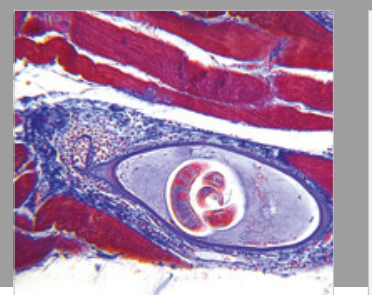

Gastroenterology Research and Practice

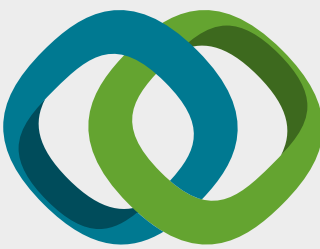

\section{Hindawi}

Submit your manuscripts at

www.hindawi.com
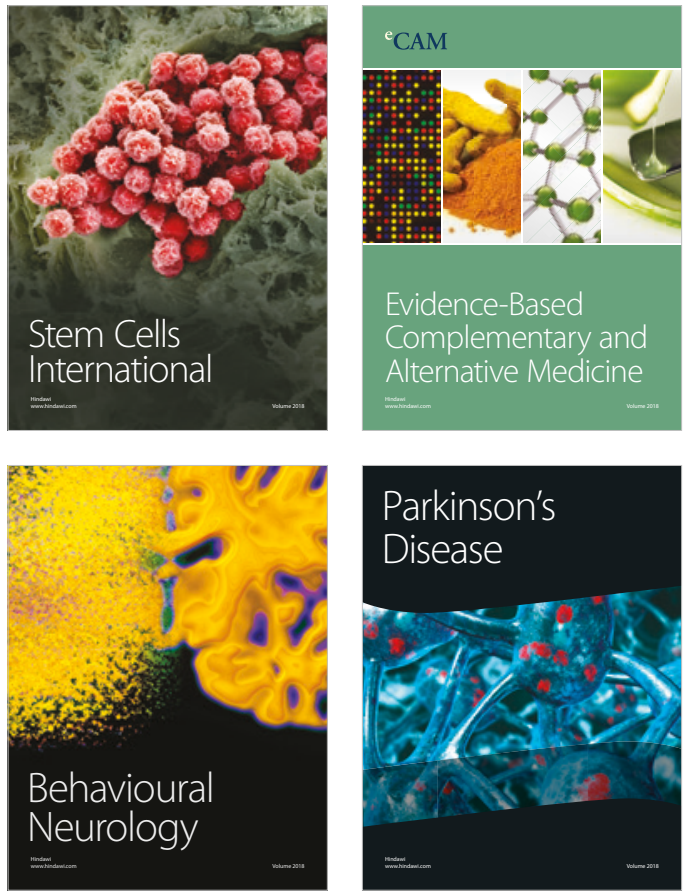

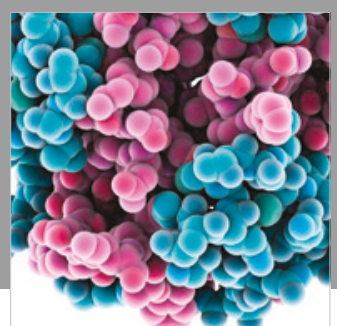

ournal of

Diabetes Research

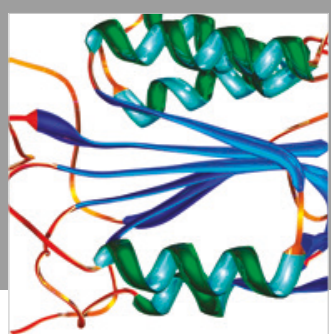

Disease Markers
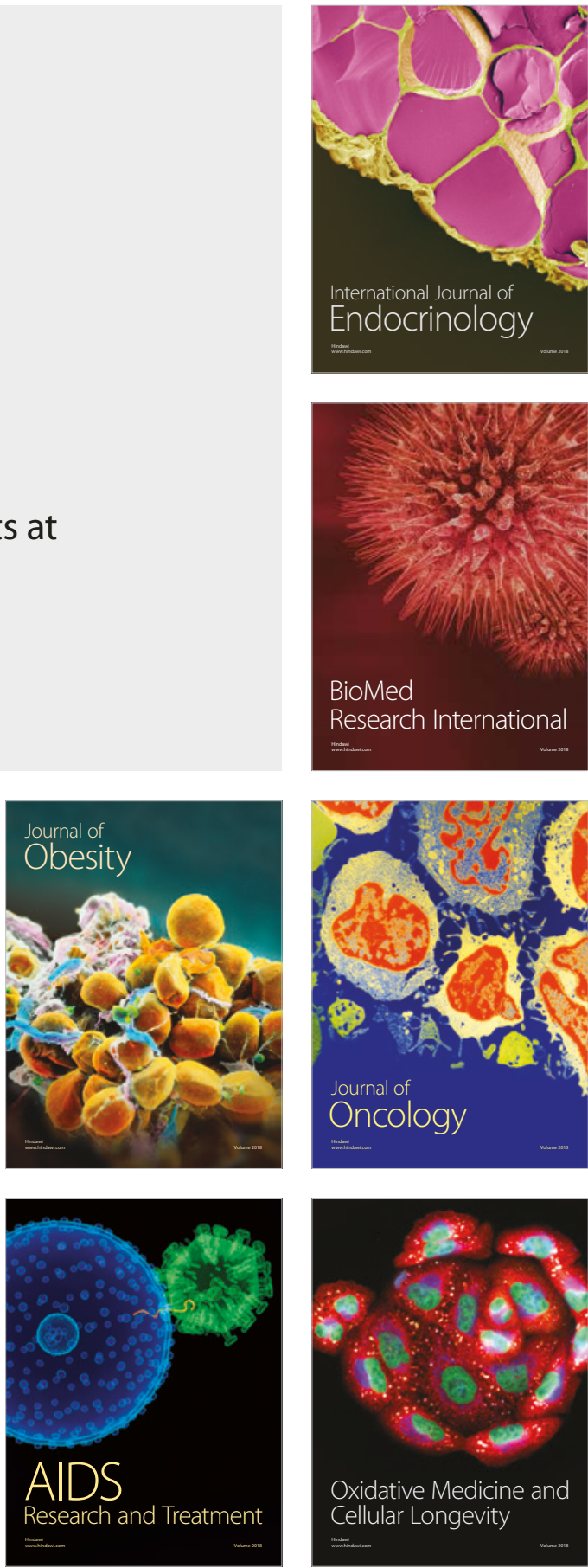